\title{
Clinical research in NSW: its role in HIV care and prevention
}

\author{
Damien V. Cordery ${ }^{\mathrm{A}, \mathrm{B}}$ and David A. Cooper \\ ${ }^{\mathrm{A}}$ National Centre in HIV Epidemiology and Clinical Research, \\ The University of New South Wales \\ ${ }^{\mathrm{B}}$ Corresponding author.Email: dcordery@nchecr.unsw.edu.au
}

Identification of HIV-related seroconversion illness In the early years of the epidemic, clinical research and vigilance in NSW was responsible for identifying and describing several important conditions associated with HIV pathogenesis and treatment. Clinical researchers based at St Vincent's Hospital in Sydney provided the first detailed description of HIV-related seroconversion illness. This mononucleosis-like illness was identified in a group of recent seroconverters. ${ }^{2}$

The identification of seroconversion illness as a marker of early infection was an important finding that created opportunities in several areas of research and patient management. Acute seroconversion illness can be a useful marker leading to early diagnosis or to providing an indication of the duration of HIV infection. From a clinical perspective, a marker for early infection allowed for more stringent patient monitoring and prophylaxis against opportunistic infection in asymptomatic patients prior to severe immunological impairment and the onset of AIDS. From a research perspective, the recognition of seroconversion illness and the identification of early HIV infections provided the opportunity to study the pathogenesis of HIV infection from pre-seroconversion through to the development of AIDS, greatly enhancing the understanding of HIV infection and disease progression. In relation to public health, the identification of a previous seroconversionrelated illness in a newly diagnosed HIV patient indicates the duration of HIV infection and may narrow the timeframe in which other people may have been exposed to HIV. This makes contacting and testing potentially exposed people more accurate and timely. The identification and evaluation of patients around the time of seroconversion has also been valuable in more accurately identifying specific risk factors associated with the transmission of HIV. The identification and description of seroconversion illness were therefore important breakthroughs in the understanding and management of HIV/AIDS early in the epidemic.

\section{Introduction of antiretroviral therapy}

Researchers and clinicians in NSW have been involved in the development and clinical trials of all the previous and currently available antiretroviral drugs. This has meant that HIV patients in NSW have had access to new treatments very early, through recruitment to clinical trials. The experience gained by clinicians during these trials has 
resulted in the rapid and informed use of new drugs in their patients as soon as they are approved for use.

The introduction of combined antiretroviral therapy has made it possible to control viral replication and elicit a partial reconstitution of immune function in HIV patients. However, the widespread availability of antiretroviral therapy has also created new challenges such as the longterm management of antiretroviral therapy use, treatmentrelated toxicity and the development of drug resistance.

Ongoing research is focused on the combinations of drugs and dosing that are most efficacious for different patient groups as well as on accurately determining the ideal time to commence treatment and whether to maintain treatment in the face of side effects. The international SMART (Strategies for Management of Anti-Retroviral Therapy) trial demonstrated that interruption to treatment was detrimental to patients, with significant increases in morbidity and all-cause mortality. ${ }^{3}$ This study conclusively ended the long-running debate as to whether treatment interruption was a safe and effective strategy in HIV patients. The ongoing START trial is designed to determine whether early or deferred initiation of antiretroviral therapy results in better outcomes for patient. ${ }^{4}$

In addition to the improved outcomes for people with HIV that have resulted from the introduction of antiretroviral therapy, widespread antiretroviral therapy use has the added benefit of decreasing the overall viral load in the HIV-positive population which also reduces infectivity. The results from the SMART study have led to a shift away from intermittent therapy to constant suppressive therapy which results in a lower overall viral load and infectivity within the community.

In the area of treatment-related toxicity, researchers in NSW have provided significant contributions. Antiretroviral-related lipodystrophy, generally characterised by a loss of fat from the face and limbs and an increase in abdominal obesity, was first identified and described by clinicians at St Vincent's Hospital, Sydney. ${ }^{5}$ Further work identifying the specific drugs responsible, elucidating the mechanisms that lead to lipodystrophy and evaluating methods for alleviating or reversing lipodystrophy have been a priority of NSW research in the antiretroviral treatment era.

\section{The antiretroviral therapy era}

HIV infection may now be managed as a chronic condition in the developed world. However this does not mean that there is a reduced role for clinical research. The main challenge in clinical research has been shifting the focus from managing opportunistic infections and the progression of AIDS to identifying and managing complications related to long-term HIV infection, such as non-AIDS related morbidity and mortality. A major focus of current research is the management and understanding of ageing in the context of HIV infection. Despite efficient viral suppression with antiretroviral treatment, the life expectancy of HIV-positive people is still reduced compared to the general population. Research is now focused on alleviating the cardiovascular disease, kidney dysfunction, neurological complications and cancers seen in HIV patients.

Factors related to HIV transmission, disease progression and immune responses to the virus have not been neglected due to the introduction of antiretroviral therapy. Several observational cohorts have been established to monitor disease progression and treatment trends in HIV-positive populations with access to antiretroviral therapy. The Australian HIV Observational Database was created in 1999 to monitor the patterns of antiretroviral therapy use relating to demographic factors and markers of HIV disease stage. ${ }^{6}$ The database is primarily focussed on collecting information about how often antiretroviral treatments are changed and the reasons for treatment changes, including adverse events. The database has allowed the trends in antiretroviral therapy usage and related adverse events to be mapped and considered in relation to demographic factors.

\section{Future directions - the development of a vaccine}

Since HIV was first identified, considerable effort and resources has been directed towards the development of a vaccine to prevent transmission of the virus. The availability of a HIV vaccine to prevent or treat infections, representing a relatively brief intervention that provides long-term protection, is clearly preferable from a medical, financial and personal perspective to the current situation of long-term continuous therapy with antiretroviral drugs. Research into vaccine development has greatly increased the knowledge of HIV pathogenesis and disease progression but unfortunately an efficacious vaccine has not been developed. Recently presented results from two international vaccine trials have influenced thinking about the progress and validity of a HIV vaccine. The first was the STEP trial: an interim analysis suggested that this vaccine enhanced rather than prevented HIV transmission and the study was subsequently stopped early in November 2007 . $^{7}$ This created some pessimism around the potential for a future HIV vaccine. More recently, a vaccine trial in Thailand involving a dosing strategy with two separate vaccine candidates (a canary pox vector and a subunit vaccine) demonstrated an approximate $30 \%$ reduction in HIV transmission in the participants who received the vaccine compared to placebo. ${ }^{8}$ Further research is required to determine the immunological factors induced by this vaccine combination, in order to enhance this response and further reduce transmission. This highlights the need for an 
improved understanding of HIV pathogenesis and immune responses, to identify not only the key mechanisms required to interrupt HIV transmission but also interventions that may induce immune responses that control HIV progression in those who already have the infection.

The recent vaccine trials also demonstrate that intensive instruction on prevention of HIV transmission, which was incorporated in these studies, does not prevent continued transmission of the virus. This adds to the argument that biomedical interventions, such as vaccines, are required in addition to education to control the HIV epidemic.

\section{Conclusion}

Clinical research will continue to contribute to the understanding of many aspects for HIV transmission, pathogenesis and treatment. National and international collaborations, established and nurtured during the course of the HIV epidemic, remain an essential part in identifying and managing the complex factors required for the current management of HIV and the potential mechanisms for the future elimination of HIV.

\section{References}

1. National Centre in HIV Epidemiology and Clinical Research. HIV/AIDS, viral hepatitis and sexually transmissible infections in Australia Annual Surveillance Report 2009. National Centre in HIV Epidemiology and Clinical Research, The University of
New South Wales, Sydney, NSW, 2009. Available at: http:// www.nchecr.unsw.edu.au/NCHECRweb.nsf/page/Annual+ Surveillance+Reports (Cited 26 February 2010.)

2. Cooper DA, Gold J, Maclean P, Donovan B, Finlayson R, Barnes $\mathrm{TG}$ et al. Acute AIDS retrovirus infection. Definition of a clinical illness associated with seroconversion. Lancet 1985; 325: 537-40. doi:10.1016/S0140-6736(85)91205-X

3. El-Sadr WM, Lundgren JD, Neaton JD, Gordin F, Abrmas D, Arduino RC et al. CD4+ count-guided interruption of antiretroviral treatment. N Engl J Med 2006; 355: 2283-96. doi:10.1056/NEJMoa062360

4. START Trial. Available at: www.clinicaltrials.gov/ct2/show/ NCT00867048?term $=$ NCT00867048\&rank $=1$ (Cited 26 February 2010.)

5. Carr A, Samaras K, Burton S, Law M, Freund J, Chisholm DJ et al. A syndrome of peripheral lipodystrophy, hyperlipidaemia and insulin resistance in patients receiving HIV protease inhibitors. AIDS 1998; 12(7): F51-8. doi:10.1097/ 00002030-199807000-00003

6. Australian HIV Observational Database. Rates of combination antiretroviral treatment change in Australia, 1997-2000. HIV Med 2002; 3(1): 28-36. doi:10.1046/j.1464-2662.2001.00094.x

7. Buchbinder SP, Mehrotra DV, Duerr A, Fitzgerald DW, Mogg R, Li D et al. Efficacy assessment of a cell-mediated immunity HIV-1 vaccine (the STEP Study): a double-blind, randomised, placebo-controlled test-of-concept trial. Lancet 2008; 372: 1881-93. doi:10.1016/S0140-6736(08)61591-3

8. Rerks-Ngarm S, Pitisuttithum P, Nitayaphan S, Kaewkungwal J, Chiu J, Paris R et al. Vaccination with ALVAC and AIDSVAX to prevent HIV-1 infection in Thailand. N Engl J Med 2009; 361: 2209-20. doi:10.1056/NEJMoa0908492 